\title{
17th ASME Conference on Information Storage and Processing Systems at Santa Clara University, Santa Clara, CA, USA
}

\author{
R. A. de Callafon $\cdot$ F. E. Talke
}

Published online: 16 September 2009

(c) The Author(s) 2009. This article is published with open access at Springerlink.com

This Special Issue is a collection of papers that were presented at the 17th ASME Conference on Information Storage and Processing Systems (ISPS) at Santa Clara University, Santa Clara, CA, USA on June 18 and 19, 2007. To archive the papers presented at this 17th ISPS conference, all authors of papers submitted to the conference were encouraged to submit a full paper to this Special Issue in Microsystems Technologies. The papers that appear in this Special Issue have been subjected to a rigorous peer-review process in which many contributions had to be revised substantially before they could be published in this Special Issue. Obviously, this process has created a special selection of the many topics presented at the 17 th ISPS conference, making it a great privilege to host the 17 th ISPS conference and serve as guest editors for this Special Issue.

The ISPS division of the ASME is the main division working on storage systems that include disk drives, tape drives, and optical drives. The ISPS division has held their annual conference at Santa Clara University in sunny California in June of each year and provides an environment in which researchers of both academic and industrial backgrounds share their latest developments on data storage systems. Since 2003, tri-annually, the ISPS division

\footnotetext{
R. A. de Callafon ( ()

Department of Mechanical and Aerospace Engineering, Jacobs School of Engineering, UC San Diego, 9500 Gilman Drive, La Jolla, CA 92093-0411, USA e-mail: callafon@ucsd.edu

F. E. Talke

Center for Magnetic Recording Research, Jacobs School of Engineering, UC San Diego, 9500 Gilman Drive, La Jolla, CA 92093-0401, USA e-mail: ftalke@ucsd.edu
}

joins forces with the IIP (Information, Intelligent, and Precision Machines) division of the JSME (Japanese Society of Mechanical Engineers) to organize a joint conference called MIPE (Micromechatronics for Information and Precision Equipment).

The annual conference of the ISPS division of the ASME is a very unique conference that brings together academia and industry in joint sessions on topics that relate to the interdisciplinary nature of the research in data storage and processing systems. To achieve the goal of merging academic and industrial research topics, the ISPS conference is held at Santa Clara in the Silicon Valley, allowing local industrial participants to attend and contribute to the conference with little travel time. With easy international access to the Bay area, the conference also enjoys many international visitors. The ISPS conference promotes industry and academic interaction by an organization that features many interdisciplinary technical sessions with a specific application focus. At the 17th ISPS conference eight different technical sessions were organized, each with dedicated session chairs to solicit papers and contributions from both academic and industrial institutions.

The technical sessions at the 17th ISPS conference included Actuators and Suspensions with applications in actuator and (dual-stage) suspension design, dynamics and analysis; Servo Control and Servo Technology: bringing together topics on dynamics, vibrations, servo design, servo writing, tracking and seeking; Tribology and Head/ Media Interface: with contributions on friction, wear, lubrication, corrosion, contamination, thin films, contact mechanics and heat transfer. Spindle Motor and Acoustics: with contemporary issues on design, vibrations, acoustics and tribology related aspects of spindle motors; Flexible Media Mechanics and Tape Storage: illustrating the issues 
related to an active research area in tape storage with design, lateral tape motion, tape tension and media; Optical Storage and Technology: focusing on media, holographic storage and servo related aspects; Shock Analysis and Flow Induced Vibrations: addressing the practical issues of data storage due to operational and non-operational shock analysis and flow induced vibrations; Micro/Nano Technologies: showing novel design concepts, production, patterned media, MEMS, NEMS and nanotechnology in data storage applications and Image Processing and Consumer Electronics: with very specific hardware applications that include digital camera, camcorder, ink jet, thermal, laser printing and optical storage.

Due to the broad scope of the technical sessions and the promotion of academic and industrial interaction, the 17th ISPS conference had a technical program with 83 unique contributions organized in 21 sessions over 2 days. The 21 sessions were run in three parallel sessions that would relate to the eight technical session topics covered by the conference. The conference was attended by more than 150 national and international attendees. One of the many highlights of the conference was the awards dinner and an extraordinary seminar by Dr. Edward Grochowski who is a consultant and data storage technologist. His technical presentation focused on magnetic storage relating to hard disk drives, alternative storage technologies, processing technologies and in particular trends projecting the future of storage.

During the awards ceremony of the 17th ISPS conference, prices for the best industry paper, the best academic paper, and the best joint academic and industry paper were awarded. The selection process of the awards was organized by an independent award committee comprised of conference attendees and based on the contents of the extended abstract and the quality of the presentation during the conference. The best industry paper award went to M. Kazemi and A. Takuda from Hutchinson Technology Inc. for their paper entitled "An Investigation of FlowInduced Off-Track Vibration of Head Gimbal Assemblies in Hard Disk Drives". The best academic paper award went to O. N. Stamnes and R. A. de Callafon from the University of California, San Diego for their paper entitled "TimeOptimal Input Shaping For Discrete-Time LTI Systems with Application to Seek Profiles of a HDD System" and the best joint academic and industry paper award was given to R. P. Ambekar, D. B. Bogy, C. S. Bhatia and
T. Watanabe from the University of California, Berkeley, National University of Singapore, and Fuji Electric Device Technology in Japan for their two papers entitled "SliderLubricant Interactions at the Head-Disk Interface Part I: Existence of a Critical Clearance" and "Slider-Lubricant Interactions at the Head-Disk Interface Part II: Effect of Media-Type, Lube Molecular Weight and Additives".

The publication of this Special Issue, although later than originally expected due to the rigorous review process, indicates a closure to the activities related to the 17th ISPS conference. The organization of his conference and the publication of this Special Issue would not have been possible without the help of many talented and motivated people within and outside of the ASME ISPS division. Among the list of people we first would like to thank are Richard Jewett, currently retired from Imation Corp. and Prof. Steve Shen from the University of Washington, for their dedicated support as a past chair of the ISPS division and their advice on organizing the conference. Prof. Shen also initiated the idea of creating Special Issues to preserve the contributions of the annual ISPS conferences. We thank Professor Bharat Bhushan from Ohio State University for his coordination with the publisher to publish this Special Issue. Our gratitude also goes towards the 2007 ISPS Executive Committee members Neal Schirle from Hitachi Global Storage Technologies, Prof. Jen-Yuan (James) Chang currently at Massey University in New Zealand and the 2007 members at large Prof. Lin Wu currently at University of Science and Technology of China, Mr. Lin Guo, from SAE Corporation and Prof. Shozo Saegusa, from Hiroshima University. We would like to thank the organizers and chairs of the technical sessions that defines the uniqueness of the ISPS conference for seeking outstanding papers for the conference. They have been invaluable in making the 17th ISPS conference a success. Last, but not least, we sincerely thank all authors and reviewers for contributing their time and effort in writing and evaluating the papers for this Special Issue. Your efforts and patience have made this collection worth the wait.

Open Access This article is distributed under the terms of the Creative Commons Attribution Noncommercial License which permits any noncommercial use, distribution, and reproduction in any medium, provided the original author(s) and source are credited. 